\title{
A Novel Scoring System for Prediction of Disease Severity in COVID-19
}

\section{OPEN ACCESS}

Edited by:

Slobodan Paessler,

The University of Texas Medical

Branch at Galveston, United States

Reviewed by:

Song Yang

Capital Medical University, China

Estanislao Nistal-Villan,

San Pablo CEU University, Spain

*Correspondence:

Yonghong Zhang

13810108505@163.com

Ronghua Jin

jin_eagle@yahoo.com

†These authors have contributed equally to this work

Specialty section:

This article was submitted to Virus and Host,

a section of the journal

Frontiers in Cellular and Infection Microbiology

Received: 09 April 2020

Accepted: 26 May 2020

Published: 05 June 2020

Citation:

Zhang C, Qin L, Li K, Wang Q, Zhao Y, Xu B, Liang L, Dai Y, Feng Y, Sun J,

Li X, Hu Z, Xiang H, Dong T, Jin R and Zhang Y (2020) A Novel Scoring System for Prediction of Disease

Severity in COVID-19.

Front. Cell. Infect. Microbiol. 10:318. doi: 10.3389/fcimb.2020.00318
Chi Zhang ${ }^{1+}$, Ling Qin ${ }^{1+}$, Kang $\mathrm{Li}^{1+}$, Qi Wang ${ }^{1}$, Yan Zhao ${ }^{1}$, Bin $\mathrm{Xu}^{1}$, Lianchun Liang ${ }^{1}$, Yanchao Dai ${ }^{1}$, Yingmei Feng ${ }^{1}$, Jianping Sun ${ }^{1}$, Xuemei $\mathrm{Li}^{1}$, Zhongjie Hu ${ }^{1}$, Haiping Xiang ${ }^{1}$, Tao Dong ${ }^{2,3}$, Ronghua Jin ${ }^{1 *}$ and Yonghong Zhang ${ }^{1,2 *}$

${ }^{1}$ Beijing You'an Hospital, Capital Medical University, Beijing, China, ${ }^{2}$ Chinese Academy of Medical Science Oxford Institute (COI), University of Oxford, Oxford, United Kingdom, ${ }^{3}$ MRC Human Immunology Unit, MRC Weatherall Institute of Molecular Medicine, University of Oxford, Oxford, United Kingdom

Background: A novel enveloped RNA beta coronavirus, Corona Virus Disease 2019 (COVID-19) caused severe and even fetal pneumonia in China and other countries from December 2019. Early detection of severe patients with COVID-19 is of great significance to shorten the disease course and reduce mortality.

Methods: We assembled a retrospective cohort of 80 patients (including 56 mild and 24 severe) with COVID-19 infection treated at Beijing You'an Hospital. We used univariable and multivariable logistic regression analyses to select the risk factors of severe and even fetal pneumonia and build scoring system for prediction, which was validated later on in a group of 22 COVID-19 patients.

Results: Age, white blood cell count, neutrophil, glomerular filtration rate, and myoglobin were selected by multivariate analysis as candidates of scoring system for prediction of disease severity in COVID-19. The scoring system was applied to calculate the predictive value and found that the percentage of ICU admission (20\%,6/30) and ventilation (16.7\%, $5 / 30)$ in patients with high risk was much higher than those $(2 \%, 1 / 50 ; 2 \%, 1 / 50)$ in patients with low risk $(p=0.009 ; p=0.026)$. The AUC of scoring system was 0.906 , sensitivity of prediction is $70.8 \%$, and the specificity is $89.3 \%$. According to scoring system, the probability of patients in high risk group developing severe disease was 20.24 times than that in low risk group.

Conclusions: The possibility of severity in COVID-19 infection predicted by scoring system could help patients to receiving different therapy strategies at a very early stage.

Topic: COVID-19, severe and fetal pneumonia, logistic regression, scoring system, prediction.

Keywords: logistic regression, severity pneumonia, COVID-19, retrospective cohort, prediction scoring system

\section{INTRODUCTION}

A cluster of cases of acute respiratory illness with unknown etiology was reported in Wuhan City, Hubei Province of China from December 2019 (Chen et al., 2020). The pathogen was identified as a novel enveloped RNA beta coronavirus by the Chinese Center for Disease Control and Prevention (CDC) (Wu et al., 2020), and was designated as severe acute respiratory syndrome 
coronavirus 2 (SARS-CoV-2) (Zhu et al., 2020). The World Health Organization (WHO) declared the novel coronavirus disease, COVID-19; a public health emergency of international concern, and by 11 March 2020, the COVID-19 outbreak was declared a global pandemic. According to Coronavirus disease 2019 (COVID-19) situation report from WHO, totally 191,127 cases of patients were laboratory confirmed and amongst them 7,807 patients died by 18th March 2020 (Liu T. et al., 2020; World Health Organization, 2020).

Infection in the majority of people is mild, with common clinical characteristics including fever, cough, and sputum. Some infected patients also reported gastrointestinal symptoms including vomiting and diarrhea (Perlman and Netland, 2009; Fehr and Perlman, 2015). Dyspnea and/or hypoxemia occurred after 1 week, with $50 \%$ of severe patients quickly progressing to acute respiratory distress syndrome, septic shock, refractory metabolic acidosis, coagulation disorders, and multiorgan failure, even life-threatening (China National Health Commission, 2020). However, there is still no clear critical predictive factors and models to prognosticate the severity of the disease. This article intends to conduct a group study of 80 patients with COVID-19 infection in a tertiary teaching hospital specializing on infectious diseases to screen for critical factors related to the disease and establish a predictive model for disease severity. Early detection of severe patients with COVID19 is of great significance to shorten the disease course and reduce mortality.

\section{MATERIALS AND METHODS}

\section{Study Population}

Patients were recruited from Beijing You'an Hospital, Capital Medical University, Beijing. A discovery cohort (80 cases) was setup between January 2020 and February 2020 and a validation group (22 cases) was setup from March to April of 2020. All participants were hospitalized patients with laboratory-confirmed COVID-19. Their clinical data was collected from Electronic Medical Record System (EMRS), Laboratory Information System (LIS) and Picture Archiving and Communication System (PACS). The study was approved by the Institutional Review Board of Beijing You'an Hospital.

\section{Clinical Definitions}

COVID-19 was diagnosed according to the diagnosis and treatment of coronavirus disease 2019 (COVID-19) recommended by the National Health Commission of China (China National Health Commission, 2020). The laboratoryconfirmed patient was defined as a positive result on high throughput sequencing or real-time reverse-transcriptasepolymerase-chain-reaction (RT-PCR) assay of nasal and pharyngeal swab specimens. The degree of severity was divided as mild infection and severe infection. Severe infection was defined as COVID-19 confirmed patients with one of conditions: respiratory distress with $\mathrm{RR}>30 / \mathrm{min}$; Blood oxygen saturation $<93 \%$; arterial oxygen partial pressure $\left(\mathrm{PaO}_{2}\right)$ /Fraction of inspired $\mathrm{O}_{2}\left(\mathrm{FiO}_{2}\right)<300 \mathrm{mmHg}$; respiratory failure with mechanical ventilation; shock; or other organ failures need intensive care in ICU. Initial stage of COVID-19 infection was defined as patients during their first week of infection only with the common clinical characteristics, such as fever, cough, sputum, vomit, and diarrhea.

\section{Treatment Procedure and End-Point of Observation}

All of patients received standard therapy according to the "Diagnosis and Treatment of Coronavirus Disease 2019" guidelines recommended by the National Health Commission of China (China National Health Commission, 2020). The observed end-point was defined as recovery or death in 28 days in hospital.

\section{Clinical Observed Variables}

A total of 48 indicators were collected from the candidates at the initial stage of COVID-19 infection, including age, gender, pre-existing conditions (respiratory disease, cardiac disease, hypertension, hyperlipemia, diabetes, kidney disease, liver disease, post-operative, and more than two kinds of diseases), presenting symptoms (fever, cough, expectoration, vomit, and diarrhea). Laboratory detections at the initial stage of COVID-19 infection included $\mathrm{pH}$, partial pressure of carbon dioxide $\left(\mathrm{PCO}_{2}\right)$, partial pressure of oxygen $\left(\mathrm{PO}_{2}\right)$, blood oxygen saturation $\left(\mathrm{SaO}_{2}\right)$, white blood cell count (WBC), hemoglobin (HGB), platelet count (PLT), absolute value of lymphocyte (LYM), absolute value of monocyte (MONO), absolute value of neutrophil (NEU), lymphocyte percentage (LYM\%), neutrophil percentage (NEU\%), ratio of neutrophil to lymphocyte (NLR), prothrombin time (PT), prothrombin activity (PTA), fibrinogen content (FIB), procalcitonin (PCT), c-reactive protein (CRP), alanine aminotransferase (ALT), aspartate aminotransferase (AST), total bilirubin (TBIL), albumin (ALB), creatinine (Cr), glomerular filtration rate (GFR), carbon dioxide combining power $(\mathrm{CO} 2 \mathrm{CP})$, creatine kinase $(\mathrm{CK})$, creatine kinase isoenzyme-MB (CK-MB), myoglobin, troponin, and lactic acid. Computerized Tomography (CT) imaging was employed to evaluate the ground-glass opacity (GGO).

\section{Statistical Analysis}

Statistical analysis of the categorical data was performed using the Chi-square test. Fisher's exact test was used since the Chi-square approximation might not hold for the relatively small sample size. Student's $t$-test was used to compare continuous values between mild and severe infection groups in which case data were normally distributed (evaluated with Kolmogorov-Smirnov test), and non-parametric $t$-test (MannWhitney test) was used when data were not normally distributed. The univariate and multivariate logistic regression analysis of variables potentially associated with severity of COVID19 infection. The optimal cutoff values were calculated in accordance with the receiver operating characteristic curves and Youden's index. The prediction value of scoring system was determined by the area under the curve (AUC). Statistical test differences were considered significant if the $P$-values were $<0.05$. Analyses were performed with SPSS software v 25.5 (IBM, NY, USA). 
TABLE 1 | Patient demographics and clinical phenotype.

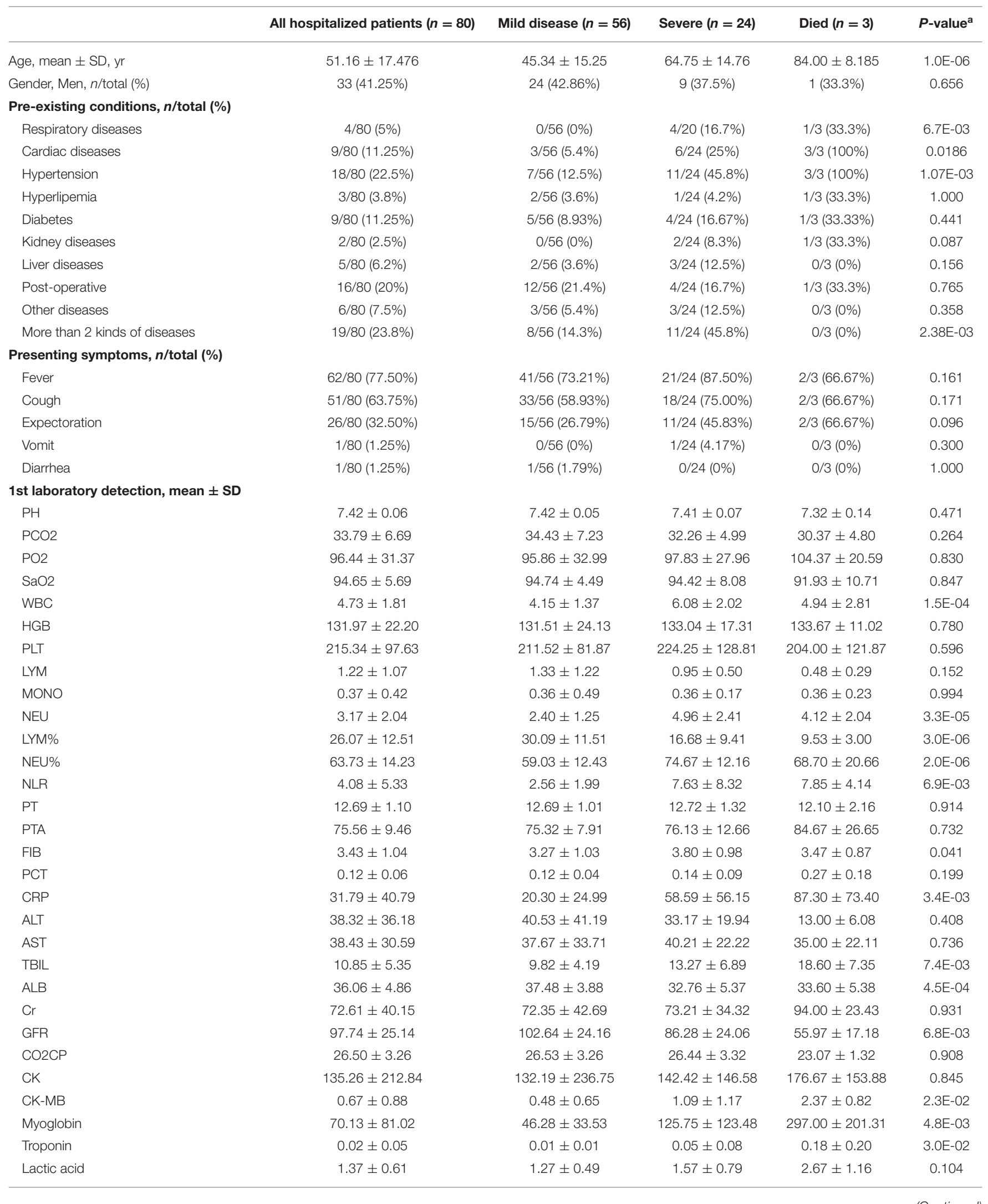


TABLE 1 | Continued

\begin{tabular}{|c|c|c|c|c|c|}
\hline & All hospitalized patients $(n=80)$ & Mild disease $(n=56)$ & Severe $(n=24)$ & Died $(n=3)$ & $P$-value ${ }^{a}$ \\
\hline \multicolumn{6}{|l|}{ Imaging of CT scan, $n /$ total (\%) } \\
\hline GGO & $74 / 80$ (92.5\%) & $51 / 56(91.1 \%)$ & 23/24 (95.8\%) & $3 / 3(100 \%)$ & 0.663 \\
\hline \multicolumn{6}{|c|}{ DIAGNOSIS MARKERS OF SEVERITY } \\
\hline \multicolumn{6}{|l|}{ Physiological variables, median (IQR) } \\
\hline $\mathrm{RR}$ & $20(20-21)$ & $20(20-20)$ & $21(20-24.75)$ & $23(20-25)$ & 2.03E-04 \\
\hline ICU admission, $n /$ total (\%) & $7 / 80(8.75 \%)$ & $0 / 56(0 \%)$ & $7 / 24(29.17 \%)$ & $3 / 3(100 \%)$ & $1.09 \mathrm{E}-04$ \\
\hline Mechanical ventilation, $n /$ total (\%) & $6 / 80(7.5 \%)$ & $0 / 56(0 \%)$ & $6 / 24(25.00 \%)$ & 3/3 (100\%) & 4.48E-04 \\
\hline 28 days mortality, $n /$ total (\%) & $3 / 80(3.75 \%)$ & $0 / 56(0 \%)$ & $3 / 24$ (12.5\%) & $3 / 3(100 \%)$ & 0.025 \\
\hline
\end{tabular}

$P C O^{2}$, partial pressure of carbon dioxide; $P \mathrm{O}_{2}$, partial pressure of oxygen; SaO2, blood oxygen saturation; WBC, white blood cell count; HGB, hemoglobin; PLT, platelet count; LYM, absolute value of lymphocyte; MONO, absolute value of monocyte; NEU, absolute value of neutrophil; LYM\%, lymphocyte percentage; NEU\%, neutrophil percentage; NLR, ratio of neutrophil to lymphocyte; PT, prothrombin time; PTA, prothrombin activity; FIB, fibrinogen content; PCT, procalcitonin; CRP, c-reactive protein; ALT, alanine aminotransferase; AST, aspartate aminotransferase; TBIL, total bilirubin; ALB, albumin; Cr, creatinine; GFR, glomerular filtration rate; CO2CP, carbon dioxide combining power; CK, creatine kinase; CK-MB, creatine kinase isoenzyme-MB; CT, Computerized Tomography; GGO, ground-glass opacity; RR, respiratory rate; P/F, PaO2/FiO2; ICU, intensive care unit.

${ }^{a} p$-values comparing severe and mild infection patients were calculated by Chi-square test and Fisher's exact test. Student's $t$-test was used where data were normally distributed (evaluated with Kolmogorov-Smirnov test), and non-parametric t-test (Mann-Whitney test) was used when data were not normally distributed. Statistical test differences were considered significant if the $P$-values were $<0.05$.

\section{RESULTS}

\section{Clinical and Laboratory Characteristics of Discovery Cohort}

Eighty hospitalized patients with laboratory-confirmed COVID19 were recruited in the study in total, and all candidates were divided into those with "mild" and "severe" disease according to the clinical definitions from the National Health Commission of China. Mild disease $(n=56)$ was defined as those with fever, respiratory symptoms and pneumonia from imaging. Patients with severe disease $(n=24)$ were those with the symptoms described above, but deteriorated and developed respiratory distress or respiratory failure. Blood oxygen saturation in the patients $(24 / 24)$ in the severe group was below $93 \%$, and none of 56 patients in mild group was below $93 \%$. The ratio of arterial oxygen partial pressure $\left(\mathrm{PaO}_{2}\right)$ to Fraction of inspired $\mathrm{O}_{2}\left(\mathrm{FiO}_{2}\right)$ was $223.5 \pm 45.77 \mathrm{mmHg}$ in severe group, much lower than that in mild group $(466.7 \pm 135.6 \mathrm{mmHg}, p<0.001)$. Seven patients in severe group received intensive care in ICU, 6 patients mechanically ventilated, and among them three severely infected patients died. Demographic data are shown in Table 1.

\section{Clinical Indicators Associated With the Severity of COVID-19 Infection}

Demographic and clinical data between mild and severe group were compared. Firstly, age was found strongly associated with the severity of diseases $(45.34 \pm 15.25$ in mild vs. $64.75 \pm 14.76$ in severe group, $p=1.0 \mathrm{E}-06)$. Secondly, respiratory disease ( $p=$ $0.0067)$, cardiac disease $(p=0.0186)$, hypertension $(p=0.0011)$, and more than two comorbidities ( $p=0.0024$ ) were identified as the factors associated with the severity. Several biomarkers from the 1st laboratory detection were also identified as the potential factors related with the severity of the disease, including white blood cell count $(4.15 \pm 1.37$ in mild vs. $6.08 \pm 2.02$ in severe group, $p=1.5 \mathrm{E}-04)$, absolute value of neutrophil $(2.40 \pm 1.25$ in mild vs. $4.96 \pm 2.41$ in severe group, $p=3.3 \mathrm{E}-05)$, lymphocyte percentage $(30.09 \pm 11.51$ in mild vs. $16.68 \pm 9.41$ in severe group, $p=3.0 \mathrm{E}-06)$, neutrophil percentage $(59.03 \pm 12.43$ in mild vs. $74.67 \pm 12.16$ in severe group, $p=2.0 \mathrm{E}-06$ ), ratio of neutrophil to lymphocyte $(2.56 \pm 1.99$ in mild vs. $7.63 \pm 8.32$ in severe group, $p=6.9 \mathrm{E}-03)$, fibrinogen content $(3.27 \pm 1.03$ in mild vs. $3.80 \pm 0.98$ in severe group, $p=0.041)$, c-reactive protein $(20.30 \pm 24.99$ in mild vs. $58.59 \pm 56.15$ in severe group, $p=3.4 \mathrm{E}-03)$, total bilirubin $(9.82 \pm 4.19$ in mild vs. $13.27 \pm$ 6.89 in severe group, $p=7.4 \mathrm{E}-03)$, albumin $(37.48 \pm 3.88$ in mild vs. $32.76 \pm 5.37$ in severe group, $p=4.5 \mathrm{E}-04)$, glomerular filtration rate $(102.64 \pm 24.16$ in mild vs. $86.28 \pm 24.06$ in severe group, $p=6.8 \mathrm{E}-03)$, creatine kinase isoenzyme-MB $(0.48 \pm 0.65$ in mild vs. $1.09 \pm 1.17$ in severe group, $p=2.3 \mathrm{E}-02$ ), myoglobin (46.28 \pm 33.53 in mild vs. $125.75 \pm 123.48$ in severe group, $p$ $=4.8 \mathrm{E}-03)$, troponin $(0.01 \pm 0.01$ in mild vs. $0.05 \pm 0.08$ in severe group, $p=3.0 \mathrm{E}-02)$. There was no significant difference in presenting symptoms and imaging of CT scan during the initial stage of COVID-19 infection between mild and severe groups. Demographic and clinical data are shown in Table 1.

\section{Scoring System for Prediction of Disease Severity in COVID-19}

The factors associated with severity of COVID-19 in Table 1 were analyzed by univariate and multivariate logistic regression analysis. Age, pre-existing conditions (cardiac disease, hypertension, and more than two comorbidities), and 1st Laboratory detection (WBC, NEU, LYM\%, NEU\%, NLR, FIB, CRP, TBIL, ALB, GRF, CK-MB, Myoglobin, and Troponin) were identified as the predictors of the severity of disease by univariate analysis. Amongst them, age, WBC, NEU, GFR, and Myoglobin were selected by multivariate analysis as candidates of scoring system for prediction of disease severity in COVID-19 (Table 2). Each variable selected by multivariate analysis was assigned diverse scores according to their hazard ratio (HR). Patients with 
TABLE 2 | Predictive factors for the severity of COVID-19 by Logistic Regression Model.

\begin{tabular}{|c|c|c|c|c|}
\hline \multirow[t]{2}{*}{ Variables } & \multicolumn{2}{|l|}{ Univariate } & \multicolumn{2}{|c|}{ Multivariate } \\
\hline & HR (95\% Cl) & $P$-value & HR (95\% Cl) & $P$-value \\
\hline Age & $1.09(1.04-1.13)$ & $<0.001$ & $1.08(0.99-1.17)$ & 0.085 \\
\hline Respiratory diseases & 1 & 0.999 & & \\
\hline Cardiac disease & $5.89(1.33-26.01)$ & 0.019 & $0.21(0.00-22.09)$ & 0.514 \\
\hline Hypertension & $5.92(1.92-18.30)$ & 0.002 & $0.35(0.03-4.08)$ & 0.399 \\
\hline $\begin{array}{l}\text { More than } 2 \text { kinds } \\
\text { of diseases }\end{array}$ & $5.08(1.69-15.22)$ & 0.004 & $7.33(0.37-146.07)$ & 0.192 \\
\hline WBC & $1.99(1.41-2.81)$ & $<0.001$ & $1.88(1.10-3.19)$ & 0.021 \\
\hline Neutrophil & $2.41(1.59-3.64)$ & $<0.001$ & $1.72(1.02-2.89)$ & 0.042 \\
\hline LYM\% & $0.88(0.82-0.94)$ & $<0.001$ & $1.07(0.94-1.22)$ & 0.285 \\
\hline NEU\% & $1.12(1.06-1.18)$ & $<0.001$ & $1.16(0.94-1.43)$ & 0.161 \\
\hline NLR & $1.55(1.18-2.03)$ & 0.001 & $1.17(0.85-1.60)$ & 0.336 \\
\hline Fib & $1.64(1.01-2.67)$ & 0.045 & 0.52 (0.09-3.03) & 0.470 \\
\hline CRP & $1.03(1.01-1.04)$ & 0.001 & $1.01(0.97-1.11)$ & 0.574 \\
\hline TBIL & $1.14(1.02-1.26)$ & 0.019 & $0.89(0.71-1.13)$ & 0.331 \\
\hline ALB & $0.80(0.70-0.90)$ & $<0.001$ & $0.87(0.66-1.14)$ & 0.322 \\
\hline GFR & $0.97(0.96-0.99)$ & 0.013 & $1.05(0.99-1.11)$ & 0.096 \\
\hline CK-MB & $2.30(1.19-4.42)$ & 0.013 & $1.22(0.18-8.43)$ & 0.844 \\
\hline Myoglobin & $1.02(1.01-1.04)$ & 0.003 & $1.02(0.99-1.04)$ & 0.094 \\
\hline Troponin & / & 0.001 & / & 0.670 \\
\hline
\end{tabular}

HR, hazard ratio; Cl, confidence interval; WBC, white blood cell count; LYM\%, lymphocyte percentage; NEU\%, neutrophil percentage; NLR, ratio of neutrophil to lymphocyte; FIB, fibrinogen content; CRP, c-reactive protein; TBIL, total bilirubin; ALB, albumin; GFR, glomerular filtration rate; $C K-M B$, creatine kinase isoenzyme-MB.

The univariate and multivariate logistic regression analysis was employed to select variables potentially associated with severity of COVID-19 infection. Statistical test differences were considered significant if the $P$-values were $<0.1$. And the $P$-value was highlighted in bold if the difference is significant.

age above 59 years old were assigned a score of 1 ; and the level of WBC above 6.09, the value of neutrophil above 2.89 were given score of 2; GFR below 103.75 and myoglobin above 43 were assigned score 1 . Finally, a scoring system was designed, which ranged from 0 to 7 by calculating each patient's score. Individuals with scores of $0-4$ were defined to be at low risk of severity, and 5-7 at high risk (Table 3 ).

\section{Predictive Value and Validation of Scoring System to the Severity of COVID-19}

The scoring system was brought into the cohort to calculate the predictive value and found that the percentage of ICU admission $(20 \%, 6 / 30)$ and ventilation $(16.7 \%, 5 / 30)$ in patients with high risk was much higher than those $(2 \%, 1 / 50 ; 2 \%, 1 / 50)$ in patients with low risk $(p=0.009 ; p=0.026)$. The scoring system was then used to evaluate the accuracy of prediction in severity and found that the AUC is 0.906 (Figure 1A), sensitivity of prediction is $70.8 \%$, and the specificity is $89.3 \%$. The probability of patients in high risk group developing severe disease was 20.24 times than that in low risk group ( $p=1.0 \mathrm{E}-06$, Table 4$)$. In addition, another 22 patients with COVID-19 were recruited from March to April of 2020 in the validation cohort. Amongst them, 18 patients were diagnosed as "mild" disease and 4 patients with "severe" disease. The variables from scoring system, including age, WBC, NEU, GFR, and Myoglobin were collected and the patients were divided into two groups (high risk vs. low risk) according
TABLE 3 | Scoring system for prediction of disease severity in COVID-19.

\begin{tabular}{lll}
\hline Variables & HR & Score \\
\hline Age & 1.08 & \\
$<59$ & & 0 \\
$\geq 59$ & 1.88 & 1 \\
WBC & & \\
$<6.09$ & & 0 \\
$\geq 6.09$ & 1.72 & 2 \\
Neutrophil & & \\
$<2.89$ & & 0 \\
$\geq 2.89$ & 1.05 & 2 \\
GFR & & \\
$<103.75$ & & 1 \\
$\geq 103.75$ & 1.02 & 0 \\
Myoglobin & & \\
$<43$ & & 0 \\
$\geq 43$ & & 1 \\
Low risk & & $0-4$ \\
High risk & & $5-7$ \\
\hline
\end{tabular}

$H R$, hazard ratio; WBC, white blood cell count; GFR, glomerular filtration rate.

TABLE 4 | Predictive value of scoring system to the severity of COVID-19.

\begin{tabular}{lcccc}
\hline Variables & Low risk & High risk & OR & $\boldsymbol{P}$-value \\
\hline ICU & $56 / 1$ & $17 / 6$ & 19.77 & 0.007 \\
Ventilation & $56 / 1$ & $18 / 5$ & 15.56 & 0.015 \\
severity & $50 / 7$ & $6 / 17$ & 20.24 & $1.0 \mathrm{E}-6$
\end{tabular}

OR, odds ratio; ICU, intensive care unit.

The prediction value of scoring system was determined by the area under the curve (AUC). Statistical test differences were considered significant if the $P$-values were $<0.05$.

to the scoring system. The accuracy of prediction in severity was evaluated and found that the AUC is 0.958 , sensitivity of prediction is $100 \%$, and the specificity is $88.9 \%$ (Figure 1B).

\section{DISCUSSION}

COVID 19 is a novel disease which has spread throughout the world and resulted in over seven thousand deaths worldwide in a few months. Most patients had mild symptoms with only $6.1 \%$ of patients progressing to severe disease requiring admission to ICU or the use of mechanical ventilation (Guan et al., 2020). There is an urgent need to find a simple and precise tool to predict the development of severity in COVID-19 infection at the early stage of disease (Wynants et al., 2020).

In the current study, we calculated a novel scoring system which could help predict the severity of COVID-19 infection from patient characteristics and clinical parameters collected on the first day of presentation to hospital. Although several factors, for example, age and NLR (Gong et al., 2020; Liu J. et al., 2020; Wang et al., 2020; Yang et al., 2020; Zhou et al., 2020) have previously been reported to be associated with the incidence of severe illness, we are the first to use scoring system to classify high and low risk of severity. We found that $63.33 \%$ of patients in the high-risk group developed severe infection, compared with only $10 \%$ of patients in low-risk group, which indicated that 

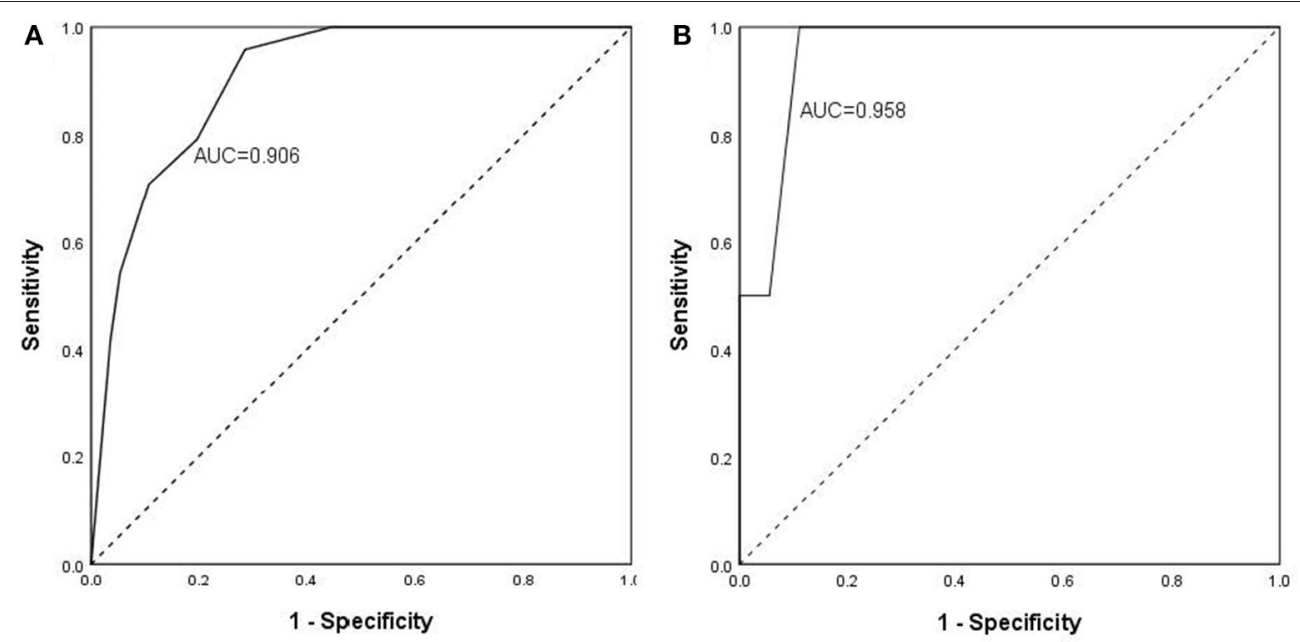

FIGURE 1 | Predictive value and validation of scoring system to the severity of COVID-19. The scoring system was brought into the discovery cohort (A) to calculate the predictive value and found that the accuracy of prediction in severity. AUC is 0.906 , sensitivity of prediction is $70.8 \%$, and the specificity is $89.3 \%$. The scoring system was brought into the validation cohort $\mathbf{( B )}$ to calculate the predictive value and found that the accuracy of prediction in severity. AUC is 0.958 , sensitivity of prediction is $100 \%$, and the specificity is $88.9 \%$.

the hazard ratio of severity in high-risk group was 20 times of low-risk group. This will help set up different strategies for high and low risk group, which is very important for government to manage limited medical resources, also useful for patients to quell anxiety.

The second character of this scoring system is covering patients' condition, from pre-existing conditions to presenting symptoms. We found that pre-existing conditions, including respiratory disease, cardiac disease, hypertension, and more than comorbidities are risk factors strongly associated with the severity, although all of them were substituted by white blood cell count, absolute value of neutrophil, glomerular filtration rate and myoglobin in scoring system, which just indicates the importance of pre-existing conditions to the severity of COVID19 infection. Amongst the five factors in scoring system, age is the basic factor of severity, which has become consensus in recent studies in COVID-19 (Gong et al., 2020) and Severe acute respiratory syndrome (SARS) (Chan et al., 2003) and Middle East respiratory syndrome (MERS) (Arabi et al., 2017). Moreover, several pre-existing conditions which are high-risk factors were reported by Gong et al. (2020), and in this study, we also found that these pre-existing conditions strongly associated with the severity for example, cardiac disease and hypertension, while they are rejected from the scoring system, because they are age-dependent factors.

In this study, white blood cell count and absolute value of neutrophil are selected to be the biomarker for predict the progress of the disease. The same as the other papers published previously, our data in the paper also found that the lymphocyte percentage descend with the disease, which indicates the direct result of viral infection (Dymond, 2018; Qin et al., 2019, 2020; Li et al., 2020; Liu Z. et al., 2020). And more interesting, we also found that the higher of white blood cell count and absolute value of neutrophil, the higher risk of severity, which give us a clue that abnormal virus-immune response cross talk in the early stage might affect the outcome of the disease (da Silva-Malta et al., 2017; Abd El-Kader and Al-Jiffri, 2018).

In addition, the biomarkers used in the scoring system are common and easily obtainable in an early stage of the disease (Havrilesky et al., 2008; Matthews et al., 2018). White blood cell count, absolute value of neutrophil, GFR and myoglobin are routine clinical detection in hospital, which could be get on the first day of hospital admission. The availability of these biomarkers indicates this scoring system could be used in an outpatient setting to classify patients in high or low risk of severity and receiving different therapy strategies.

In conclusion, our data clearly present a simple and precise scoring system to predict the possibility of severity in COVID-19 infection. Age, white blood cell count and pre-existing conditions could help calculate the score and further classify the risk of disease severity. Whilst the convenience of this scoring system is very important for current therapy during the period of pandemic of COVID-19 infection, further validation in large cohort is required.

\section{DATA AVAILABILITY STATEMENT}

The raw data supporting the conclusions of this article will be made available by the authors, without undue reservation.

\section{ETHICS STATEMENT}

The studies involving human participants were reviewed and approved by Institutional Review Board of Beijing You'an Hospital. The patients/participants provided their written informed consent to participate in this study. Written informed consent was obtained from the individual(s) for the publication of any potentially identifiable images or data included in this article. 


\section{AUTHOR CONTRIBUTIONS}

YZhang, RJ, and TD conceptualized and designed the study. KL, LQ, YZhang, YZhao, and QW analyzed the data. CZ, LQ, KL, BX, LL, YD, YF, JS, XL, ZH, and HX helped with the clinical sample and data collection. YZhang and TD wrote, reviewed and revised the manuscript.

\section{FUNDING}

This work was supported by the Beijing Natural Science Foundation (7191004 and 7202069), Beijing Municipal Science

\section{REFERENCES}

Abd El-Kader, S. M., and Al-Jiffri, O. H. (2018). Impact of weight reduction on selected immune system response among Hepatitis C virus Saudi patients. Afr. Health Sci. 18, 417-427. doi: 10.4314/ahs.v18i2.27

Arabi, Y. M., Balkhy, H. H., Hayden, F. G., Bouchama, A., Luke, T., Baillie, K., et al. (2017). Middle east respiratory syndrome. N. Engl. J. Med. 376, 584-594. doi: 10.1056/NEJMsr1408795

Chan, J. W., Ng, C. K., Chan, Y. H., Mok, T. Y., Lee, S., Chu, S. Y., et al. (2003). Short term outcome and risk factors for adverse clinical outcomes in adults with severe acute respiratory syndrome (SARS). Thorax 58, 686-689. doi: 10.1136/thorax.58.8.686

Chen, N., Zhou, M., Dong, X., Qu, J., Gong, F., Han, Y., et al. (2020). Epidemiological and clinical characteristics of 99 cases of 2019 novel coronavirus pneumonia in Wuhan, China: a descriptive study. Lancet 395, 507-513. doi: 10.1016/S0140-6736(20)30211-7

China National Health Commission (2020). The New Coronavirus Pneumonia Diagnosis and Treatment Plan (Trial version 6).

da Silva-Malta, M. C. F., Sales, C. C., Guimarães, J. C., de Cássia Gonçalves, P., Chaves, D. G., Santos, H. C., et al. (2017). The Duffy null genotype is associated with a lower level of CCL2, leukocytes and neutrophil count but not with the clinical outcome of HTLV-1 infection. J. Med. Microbiol. 66, 1207-1216. doi: 10.1099/jmm.0.000539

Dymond, T. (2018). The effects of viral infection on lymphocyte metabolism: a new perspective on disease characterization. Viral Immunol. 31, 278-281. doi: 10.1089/vim.2017.0194

Fehr, A. R., and Perlman, S. (2015). "Coronaviruses: an overview of their replication and pathogenesis," in Coronaviruses: Methods and Protocols, eds H. J. Maier, E. Bickerton, and Britton P (New York, NY: Springer New York), 1-23.

Gong, J., Ou, J., Qiu, X., Jie, Y., Chen, Y., Yuan, L., et al., (2020). A Tool for Early Prediction of Severe Coronavirus Disease 2019 (COVID-19): A Multicenter Study Using the Risk Nomogram in Wuhan and Guangdong, China. Clin. Infect. Dis. ciaa443. doi: 10.1093/cid/ciaa443

Guan, W.-J., Ni, Z.-Y., Hu, Y., Liang, W. H., Ou, C. Q., He, J. X., et al. (2020). Clinical characteristics of coronavirus disease 2019 in China. N. Engl. J. Med. 382, 1708-1720. doi: 10.1056/NEJMoa2002032

Havrilesky, L. J., Whitehead, C. M., Rubatt, J. M., Cheek, R. L., Groelke, J., He, Q., et al. (2008). Evaluation of biomarker panels for early stage ovarian cancer detection and monitoring for disease recurrence. Gynecol. Oncol. 110, 374-382. doi: 10.1016/j.ygyno.2008.04.041

Li, T., Qi, W., Duanyang, Z., Ding, J., Huang, Q., Tang, Y. Q., et al. (2020). Lymphopenia predicts disease severity of COVID-19: a descriptive and predictive study. Signal Transduct. Target. Ther. 5, 1-3. doi: 10.1038/s41392-019-0089-y

Liu, J., Liu, Y., Xiang, P., Pu, L., Xiong, H., Li, C., et al. (2020). Neutrophil-tolymphocyte ratio predicts critical illness patients with 2019 coronavirus disease in the early stage. J. Transl. Med. 18:206.

Liu, T., Hu, J., Kang, M., Lin, L., Zhong, H., Xiao, J., et al. (2020). Transmission dynamics of 2019 novel coronavirus (2019-nCoV). bioRxiv [Preprint]. doi: 10.1101/2020.01.25.919787

Liu, Z., Long, W., Tu, M., Chen, S., Huang, Y., Wang, S., et al. (2020). Lymphocyte subset $(\mathrm{CD} 4+, \mathrm{CD} 8+)$ counts reflect the severity of infection
\& Technology Commission (Z171100001017078), Capital health development research project (2020-1-2182), Beijing municipal administration of hospitals (DFL20181701 and ZYLX201711), Beijing Key Laboratory (BZ0373), China primary healthcare foundation You'An foundation of liver disease and aids scientific research project of You'an Hospital, CCMU (YNKTTS20180117), and Chinese Academy of Medical Sciences (CAMS) Innovation Fund for Medical Sciences (CIFMS), China (grant number: 2018-I2M-2-002); TD is supported by Medical Research Council (MRC), UK (MR/L018942/1 and MRC Human Immunology Unit Core) and Nuffield Department of Medicine, Oxford University.

and predict the clinical outcomes in patients with COVID-19. J. infect. doi: 10.1016/j.jinf.2020.03.054. [Epub ahead of print].

Matthews, D. C., Lerman, H., Lukic, A., Andrews, R. D., Mirelman, A., Wernick, M. N., et al. (2018). FDG PET Parkinson's disease-related pattern as a biomarker for clinical trials in early stage disease. NeuroImage Clin. 20, 572-579. doi: 10.1016/j.nicl.2018.08.006

Perlman, S., and Netland, J. (2009). Coronaviruses post-SARS: update on replication and pathogenesis. Nat. Rev. Microbiol. 7, 439-450. doi: $10.1038 /$ nrmicro2147

Qin, C., Zhou, L., Hu, Z., Zhang, S., Yang, S., Tao, Y., et al. (2020). Dysregulation of immune response in patients with COVID-19 in Wuhan, China. Clin. Infect. Dis. 12:ciaa248. doi: 10.2139/ssrn.3541136

Qin, L., Qiu, Z., Hsieh, E., Geng, T., Zhao, J., Zeng, X., et al. (2019). Association between lymphocyte subsets and cytomegalovirus infection status among patients with systemic lupus erythematosus: a pilot study. Medicine 98:e16997. doi: 10.1097/MD.0000000000 016997

Wang, L., He, W., Yu, X., Hu, D., Bao, M., Liu, H., et al. (2020). Coronavirus disease 2019 in elderly patients: characteristics and prognostic factors based on 4-week follow-up. J. Infect. 80, 639-645. doi: 10.1016/j.jinf.2020. 03.01

World Health Organization (2020). Coronavirus Disease 2019 (COVID-19) Situation Report.

Wu, F., Zhao, S., Yu, B., Chen, Y. M., Wang, W., Song, Z. G., et al. (2020). A new coronavirus associated with human respiratory disease in China. Nature 579, 265-269. doi: 10.1038/s41586-0202008-3

Wynants, L., Van Calster, B., Bonten, M. M. J., Collins, G. S., Debray, T. P. A., De Vos, M., et al. (2020). Prediction models for diagnosis and prognosis of covid-19 infection: systematic review and critical appraisal. BMJ. 369:m1328. doi: 10.1136/bmj.m1328

Yang, A., Liu, J., Tao, W., and Li, H. (2020).The diagnostic and predictive role of NLR, d-NLR and PLR in COVID-19 patients. Int. Immunopharmacol. doi: 10.1016/j.intimp.2020.106504. [Epub ahead of print].

Zhou, F., Yu, T., Du, R., Fan, G., Liu, Y., Liu, Z., et al. (2020). Clinical course and risk factors for mortality of adult inpatients with COVID-19 in Wuhan, China: a retrospective cohort study. Lancet 395, 1054-1062. doi: 10.1016/S0140-6736(20)30566-3

Zhu, N., Zhang, D., Wang, W., Li, X., Yang, B., Song, J., et al. (2020). A novel coronavirus from patients with pneumonia in China, 2019. N. Engl. J. Med. 382, 727-733. doi: 10.1056/NEJMoa2001017

Conflict of Interest: The authors declare that the research was conducted in the absence of any commercial or financial relationships that could be construed as a potential conflict of interest.

Copyright (C) 2020 Zhang, Qin, Li, Wang, Zhao, Xu, Liang, Dai, Feng, Sun, Li, Hu, Xiang, Dong, Jin and Zhang. This is an open-access article distributed under the terms of the Creative Commons Attribution License (CC BY). The use, distribution or reproduction in other forums is permitted, provided the original author(s) and the copyright owner(s) are credited and that the original publication in this journal is cited, in accordance with accepted academic practice. No use, distribution or reproduction is permitted which does not comply with these terms. 